\title{
Participant Flow
}

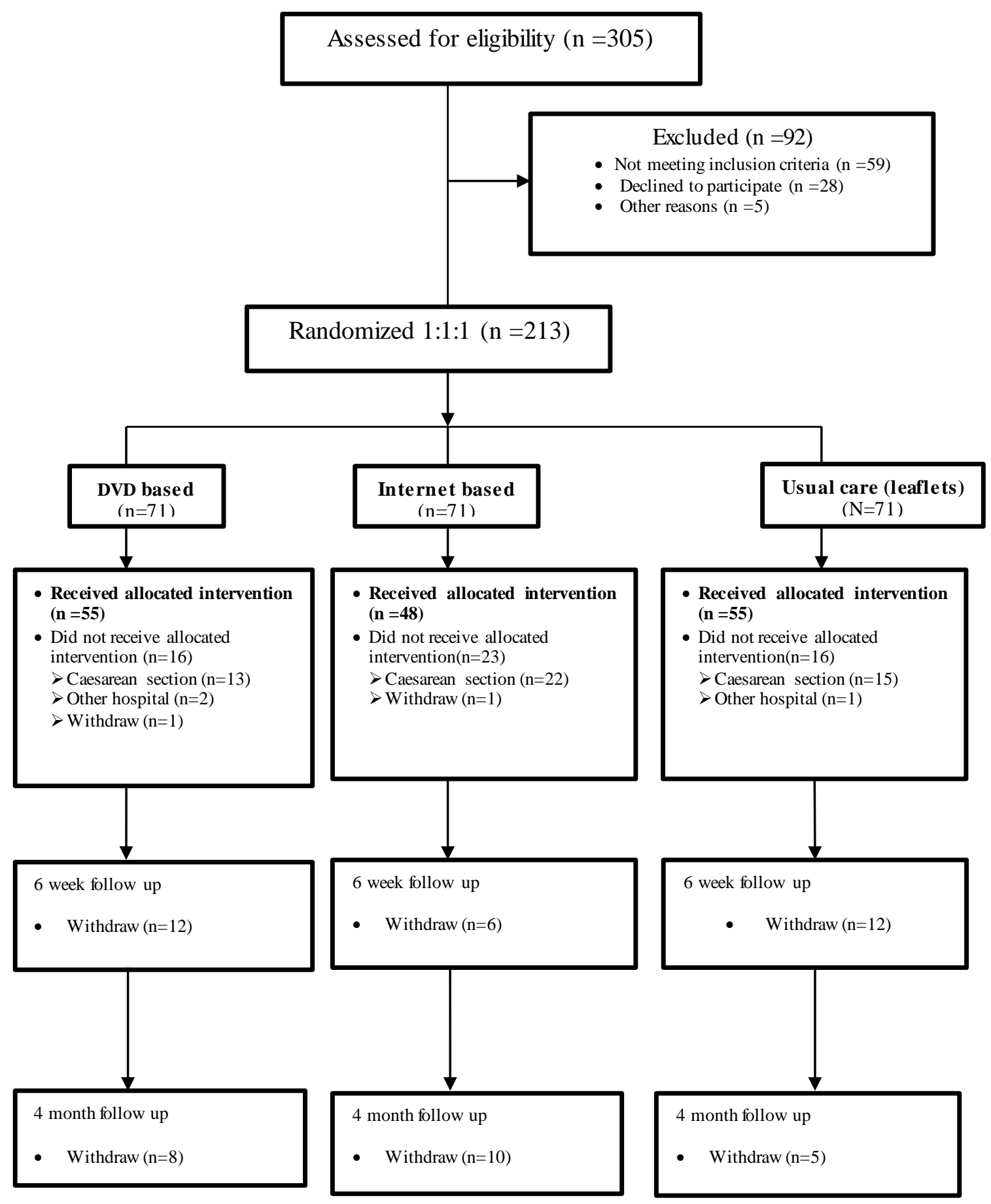

Figure 1: Study CONSORT flow diagram. 
Baseline Characteristics

Table 1: Baseline biographical profile of women per group ( $n=213)$

\begin{tabular}{|c|c|c|c|c|c|c|c|c|c|}
\hline \multirow[b]{2}{*}{ Characteristic } & \multicolumn{2}{|c|}{ All } & \multicolumn{2}{|c|}{ DVD-based } & \multicolumn{2}{|l|}{ Internet-base } & \multicolumn{2}{|c|}{ sual care } & \multirow{2}{*}{$\begin{array}{c}p \\
\text { Comparison } \\
\text { Among the } \\
\text { Groups }\end{array}$} \\
\hline & $\bar{x}(\mathrm{SD})$ & Range & $\bar{x}(\mathrm{SD})$ & Range & $\bar{x}(\mathrm{SD})$ & Range & $\bar{x}(\mathrm{SD})$ & Range & \\
\hline Age (years) & $31.5(4.8)$ & $18-40.5$ & 31.4 (4.9) & $18-40.4$ & $31.0(5.1)$ & $18-40.5$ & $32.0(4.3)$ & $20.6-40$ & 0.453 \\
\hline Height (cm) & $160.1(5.1)$ & $149-175$ & $160.4(4.8)$ & $150-171$ & $160.0(5.3)$ & $150-175$ & $159.9(5.4)$ & $149-172$ & 0.857 \\
\hline Weight (Kg) & $67.8(10.1)$ & $45-113.4$ & 67.2 (7.9) & $49-86$ & $68.8(12.3)$ & 51.5-113.4 & $67.6(9.6)$ & $45-95.4$ & 0.627 \\
\hline Gestation week & $36.5(1.6)$ & $34-40.5$ & $36.6(1.4)$ & $34.1-40.5$ & $36.2(1.6)$ & $34-40$ & $36.6(1.6)$ & 34-39.5 & 0.272 \\
\hline BMI at recruitment & $26.3(3.7)$ & $13.3-41.5$ & $26.1(3.0)$ & $20.4-33.5$ & $26.5(4.5)$ & $13.3-41.1$ & $26.4(3.5)$ & $18.7-36.1$ & 0.762 \\
\hline $\begin{array}{l}\text { BMI before } \\
\text { pregnancy }\end{array}$ & $21.3(3.1)$ & $15.7-35.3$ & $20.8(2.6)$ & $15.7-28.2$ & $21.8(3.6)$ & $15.8-35.3$ & $21.2(3.0)$ & $15.9-30.8$ & 0.166 \\
\hline
\end{tabular}


Table 2: Baseline demographical profile of women per group ( $n=213)$

\begin{tabular}{|c|c|c|c|c|c|}
\hline \multirow[b]{2}{*}{ Characteristic } & All & DVD-based & Internet-base & Usual care & \\
\hline & N (\%) & $\mathbf{N}(\%)$ & $\mathbf{N}(\%)$ & N (\%) & $\begin{array}{c}p \\
\text { Comparison } \\
\text { Among the } \\
\text { Groups }\end{array}$ \\
\hline Education & & & & & 0.154 \\
\hline secondary school & $2(0.9)$ & $1(0.5)$ & $1(0.5)$ & - & \\
\hline High school & 39 (18.3) & $8(3.8)$ & $15(7)$ & $16(7.5)$ & \\
\hline College & $32(15.0)$ & $9(4.2)$ & $8(3.8)$ & $15(7.0)$ & \\
\hline Bachelor's degree & $123(57.7)$ & $49(23)$ & $38(17.8)$ & 36 (16.9) & \\
\hline Master's degree & $17(8.0)$ & $4(1.9)$ & $9(4.2)$ & $4(1.9)$ & \\
\hline Marital status & & & & & 0.238 \\
\hline Single & $5(2.3)$ & $2(0.9)$ & $3(1.4)$ & - & \\
\hline Married & $208(97.7)$ & 69 (32.4) & 68 (31.9) & 71 (33.3) & \\
\hline $\begin{array}{l}\text { Employment } \\
\text { Status }\end{array}$ & & & & & 0.680 \\
\hline Fulltime & $154(72.3)$ & $48(22.5)$ & 51 (23.9) & $55(25.8)$ & \\
\hline Part time & $8(3.8)$ & $4(1.9)$ & $2(0.9)$ & $2(0.9)$ & \\
\hline Unemployed & $51(23.9)$ & $19(8.9)$ & $18(8.5)$ & $14(6.6)$ & \\
\hline $\begin{array}{l}\text { Exercise Before } \\
\text { Pregnancy }\end{array}$ & & & & & 0.698 \\
\hline Yes & $116(54.5)$ & 39 (18.3) & $41(19.2)$ & 36 (16.9) & \\
\hline No & $97(45.5)$ & $32(15.0)$ & $30(14.1)$ & 35 (16.4) & \\
\hline Exercise During & & & & & 0.781 \\
\hline Pregnancy & & & & & \\
\hline Yes & $118(55.4)$ & 41 (19.2) & $40(18.8)$ & 37 (17.4) & \\
\hline No & $95(44.6)$ & $30(14.1)$ & $31(14.6)$ & $34(16.0)$ & \\
\hline $\begin{array}{l}\text { Type of exercise } \\
\text { before pregnancy }\end{array}$ & & & & & 0.798 \\
\hline $\begin{array}{l}\text { To take a walk } \\
\text { over } 30 \text { minutes } \\
\text { per day }\end{array}$ & $42(36.2)$ & $13(11.2)$ & $15(12.9)$ & $14(12.0)$ & \\
\hline Brisk walking & $8(6.9)$ & $2(1.7)$ & $2(1.7)$ & $4(3.4)$ & \\
\hline Yoga & $13(11.2)$ & $4(3.4)$ & $7(6.0)$ & $2(1.7)$ & \\
\hline Aerobicexercise & 15 (12.9) & $7(6.0)$ & $4(3.4)$ & $4(3.4)$ & \\
\hline Swim & $10(8.6)$ & $4(3.4)$ & $4(3.4)$ & $2(1.7)$ & \\
\hline Gym & $16(13.7)$ & $5(4.3)$ & $6(5.1)$ & $5(4.3)$ & \\
\hline Ball exercise & $3(2.5)$ & $2(1.7)$ & $1(0.8)$ & - & \\
\hline running & $9(7.7)$ & $2(1.7)$ & $2(1.7)$ & $5(4.3)$ & \\
\hline
\end{tabular}




\section{Outcome Measures}

Primary outcome measure

Table 3: Primary Outcome: VAS in mm (0-100) of LPP at 3 days $(n=158)$ and six weeks postpartum $(n=128)$ and four months postpartum $(n=105)$

\begin{tabular}{|c|c|c|c|c|c|c|c|c|c|}
\hline \multirow[b]{2}{*}{ Characteristic } & \multicolumn{2}{|c|}{ All } & \multicolumn{2}{|c|}{ DVD-based } & \multicolumn{2}{|c|}{ Internet-base } & \multicolumn{2}{|c|}{ Usual care } & \multirow[b]{2}{*}{$\begin{array}{c}\text { P Comparison } \\
\text { Among the } \\
\text { Groups }\end{array}$} \\
\hline & $\bar{x}(\mathrm{SD})$ & Range & $\bar{x}(\mathrm{SD})$ & Range & $\bar{x}(\mathrm{SD})$ & Range & $\bar{x}(\mathrm{SD})$ & Range & \\
\hline \multicolumn{10}{|l|}{ At 3 days } \\
\hline Pain at present & $30.2(24.2)$ & $0-100$ & 38.8 (27.9) & $0-100$ & $43.4(29.4)$ & $0-100$ & $37.4(27.6)$ & $0-100$ & $0.042 *$ \\
\hline Pain at past week & $51.4(25.3)$ & $0-100$ & $44.8(24.1)$ & $0-90$ & $44.4(27.1)$ & $0-100$ & $47.0(25.6)$ & $0-100$ & 0.286 \\
\hline \multicolumn{10}{|c|}{ At six weeks postpartum } \\
\hline Pain at present & $10.4(15.2)$ & $0-60$ & $6.5(12.3)$ & $0-50$ & $10.7(14.8)$ & $0-60$ & $14.1(17.5)$ & $0-50$ & 0.064 \\
\hline Pain at past week & $16.3(18.6)$ & $0-70$ & $16.5(19.5)$ & $0-70$ & $10.6(14.3)$ & $0-50$ & $21.7(20.2)$ & $0-60$ & $0.022^{*}$ \\
\hline \multicolumn{10}{|c|}{ At four months postpartum } \\
\hline Pain at present & $4.0(10.4)$ & $0-60$ & $2.8(9.2)$ & $0-50$ & $3.2(7.1)$ & $0-30$ & $5.6(13.4)$ & $0-60$ & 0.471 \\
\hline Pain at past week & $7.1(13.5)$ & $0-70$ & $7.1(11.7)$ & $0-50$ & $4.1(9.8)$ & $0-50$ & $9.7(17.0)$ & $0-70$ & 0.229 \\
\hline
\end{tabular}

* Significant differences between the groups $(P<0.05)$ 
Table 4: DRI in $\mathrm{mm}(\mathrm{mm})$ at six weeks postpartum for three groups (mean values) ( $\mathrm{n=128}$ )

\begin{tabular}{|c|c|c|c|c|c|c|c|c|c|}
\hline \multirow[b]{2}{*}{ Characteristic } & \multicolumn{2}{|l|}{ All } & \multicolumn{2}{|l|}{ DVD-based } & \multicolumn{2}{|c|}{ Internet-base } & \multicolumn{2}{|l|}{ Usual care } & \multirow{2}{*}{$\begin{array}{l}\text { P Comparison } \\
\text { Among the } \\
\text { Groups }\end{array}$} \\
\hline & $\bar{x}(\mathrm{SD})$ & Range & $\bar{x}(\mathrm{SD})$ & Range & $\bar{x}(\mathrm{SD})$ & Range & $\bar{x}(\mathrm{SD})$ & Range & \\
\hline Dressing (without any help) & $1.28(4.87)$ & $0-30$ & $0.46(3.04)$ & $0-20$ & $1.19(4.91)$ & $0-30$ & $2.20(6.10)$ & $0-30$ & 0.250 \\
\hline Outdoor walking & $1.36(4.84)$ & $0-30$ & $0.69(3.37)$ & $0-20$ & $1.54(5.35)$ & $0-30$ & $1.86(5.56)$ & $0-30$ & 0.520 \\
\hline Climbing stairs & $2.79(7.69)$ & $0-50$ & $1.62(3.37)$ & $0-30$ & $3.04(9.34)$ & $0-50$ & $3.72(7.95)$ & $0-30$ & 0.440 \\
\hline Sitting for a long time & $8.87(14.0)$ & $0-70$ & $6.48(10.8)$ & $0-40$ & $7.61(12.6)$ & $0-50$ & $12.4(17.3)$ & $0-70$ & 0.108 \\
\hline Standing bent over a sink & $4.75(11.3)$ & $0-70$ & $2.32(7.18)$ & $0-40$ & $3.88(8.64)$ & $0-40$ & $8.02(15.7)$ & $0-70$ & 0.055 \\
\hline Carrying a bag & $4.04(9.21)$ & $0-50$ & $4.18(10.0)$ & $0-50$ & $3.69(7.89)$ & $0-35$ & $4.25(9.73)$ & $0-40$ & 0.954 \\
\hline Making a bed & $6.09(12.1)$ & $0-60$ & $5.11(10.5)$ & $0-40$ & $5.71(9.72)$ & $0-30$ & $7.44(15.4)$ & $0-60$ & 0.656 \\
\hline Running & $9.09(16.4)$ & $0-100$ & $5.81(10.9)$ & $0-40$ & $9.38(15.6)$ & $0-80$ & $12.0(20.8)$ & $0-100$ & 0.206 \\
\hline Doing light work & $3.12(7.26)$ & $0-30$ & $5.47(9.67)$ & $0-30$ & $1.16(3.90)$ & $0-20$ & $2.79(6.57)$ & $0-30$ & $0.021^{*}$ \\
\hline Doing heavy work & $14.2(19.2)$ & $0-100$ & $12.2(18.3)$ & $0-50$ & $16.1(16.3)$ & $0-60$ & $14.4(22.5)$ & $0-100$ & 0.643 \\
\hline Lifting heavy objects & $14.1(19.7)$ & $0-100$ & $12.4(20.6)$ & $0-80$ & $14.4(16.3)$ & $0-60$ & $15.5(22.2)$ & $0-100$ & 0.726 \\
\hline Participating in exercise/sport & $9.76(16.0)$ & $0-80$ & $6.39(14.7)$ & $0-80$ & $10.8(17.0)$ & $0-80$ & $12.0(16.0)$ & $0-50$ & 0.225 \\
\hline DRI total & $6.63(8.7)$ & $0-39$ & $5.26(8.4)$ & $0-28$ & $6.54(7.3)$ & $0-39$ & $8.08(10.1)$ & $0-30$ & 0.239 \\
\hline
\end{tabular}

* Significant differences between the groups $(\mathrm{P}<0.05)$ 
Table 5: DRI in $\mathrm{mm}(\mathrm{mm})$ at four months postpartum for three groups (mean values) ( $\mathrm{n=105}$ )

\begin{tabular}{|c|c|c|c|c|c|c|c|c|c|}
\hline \multirow[b]{2}{*}{ Characteristic } & \multicolumn{2}{|l|}{ All } & \multicolumn{2}{|l|}{ DVD-based } & \multicolumn{2}{|c|}{ Internet-base } & \multicolumn{2}{|l|}{ Usual care } & \multirow[b]{2}{*}{$\begin{array}{l}P \text { Comparison } \\
\text { Among the } \\
\text { Groups }\end{array}$} \\
\hline & $\bar{x}(\mathrm{SD})$ & Range & $\bar{x}(\mathrm{SD})$ & Range & $\bar{x}(\mathrm{SD})$ & Range & $\bar{x}(S D)$ & Range & \\
\hline Dressing (without any help) & $1.00(5.29)$ & $0-40$ & $0.85(5.07)$ & $0-30$ & $0.78(3.61)$ & $0-20$ & $1.31(6.64)$ & $0-40$ & 0.900 \\
\hline Outdoor walking & $0.80(4.55)$ & $0-40$ & $0.28(1.69)$ & $0-10$ & $1.09(3.96)$ & $0-20$ & $1.05(6.48)$ & $0-40$ & 0.710 \\
\hline Climbing stairs & $1.57(7.44)$ & $0-60$ & $0.85(2.84)$ & $0-10$ & $2.03(7.81)$ & $0-40$ & $1.84(9.82)$ & $0-60$ & 0.784 \\
\hline Sitting for a long time & 4.95 (11.9) & $0-70$ & $4.57(10.1)$ & $0-40$ & $2.65(7.18)$ & $0-30$ & $7.23(15.8)$ & $0-70$ & 0.272 \\
\hline Standing bent over a sink & $2.28(8.88)$ & $0-70$ & $2.00(6.32)$ & $0-30$ & $0.15(0.88)$ & $0-5$ & $4.34(13.2)$ & $0-70$ & 0.141 \\
\hline Carrying a bag & $3.47(11.2)$ & $0-70$ & $3.71(11.1)$ & $0-50$ & $1.40(4.25)$ & $0-20$ & $5.00(14.8)$ & $0-70$ & 0.411 \\
\hline Making a bed & $2.52(7.40)$ & $0-45$ & $2.28(5.46)$ & $0-20$ & $0.78(2.57)$ & $0-10$ & $4.21(10.7)$ & $0-45$ & 0.152 \\
\hline Running & $4.00(10.8)$ & $0-50$ & $2.85(7.10)$ & $0-30$ & $3.90(12.3)$ & $0-50$ & $5.13(12.4)$ & $0-45$ & 0.674 \\
\hline Doing light work & $1.00(4.06)$ & $0-30$ & $0.57(2.35)$ & $0-10$ & $1.40(5.56)$ & $0-30$ & $1.05(3.88)$ & $0-20$ & 0.704 \\
\hline Doing heavy work & $6.04(13.2)$ & $0-60$ & $6.00(13.1)$ & $0-50$ & $3.90(9.22)$ & $0-40$ & $7.89(16.1)$ & $0-60$ & 0.462 \\
\hline Lifting heavy objects & $6.52(14.3)$ & $0-60$ & $7.42(15.9)$ & $0-60$ & $2.96(8.11)$ & $0-40$ & $8.68(16.4)$ & $0-60$ & 0.228 \\
\hline Participating in exercise/sport & $3.28(9.24)$ & $0-50$ & $2.57(7.80)$ & $0-40$ & $2.65(8.03)$ & $0-40$ & $4.47(11.3)$ & $0-50$ & 0.616 \\
\hline DRI total & $3.04(6.84)$ & $0-40$ & $2.79(5.33)$ & $0-20$ & $1.86(4.72)$ & $0-23$ & $4.27(9.17)$ & $0-40$ & 0.334 \\
\hline
\end{tabular}


Table 6: Physical testing and diastasis recti at discharge $(n=158)$, six weeks ( $n=128)$ and four months postpartum ( $n=105)$ for three groups (mean values)

\begin{tabular}{|c|c|c|c|c|c|c|c|c|c|}
\hline \multirow[b]{2}{*}{ Characteristic } & \multicolumn{2}{|c|}{ All } & \multicolumn{2}{|c|}{ DVD-based } & \multicolumn{2}{|c|}{ Internet-base } & \multicolumn{2}{|c|}{ Usual care } & \multirow{2}{*}{$\begin{array}{c}P \\
\text { Comparison } \\
\text { Among the } \\
\text { Groups }\end{array}$} \\
\hline & $\bar{x}(\mathrm{SD})$ & Range & $\bar{x}(S D)$ & Range & $\bar{x}(S D)$ & Range & $\bar{x}$ (SD) & Range & \\
\hline \multicolumn{10}{|l|}{$\begin{array}{l}\text { Discharge period } \\
\text { Physical testing }\end{array}$} \\
\hline Body weight & $64.0(9.77)$ & $43.9-91.3$ & $63.1(7.90)$ & $49.2-82.1$ & $65.3(11.5)$ & $46.7-86.9$ & $63.8(9.79)$ & $43.9-91.3$ & 0.515 \\
\hline $\begin{array}{c}\text { Waist circumference } \\
\text { Diastasis recti }\end{array}$ & $92.2(9.04)$ & $59-134$ & $91.5(8.26)$ & 67-107 & $92.8(9.08)$ & 77-110 & $92.4(9.85)$ & 59-134 & 0.771 \\
\hline Umbilicus $4.5 \mathrm{~cm}$ above & $1.87(0.65)$ & $0-4$ & $1.83(0.62)$ & $0.5-3$ & $1.79(0.60)$ & $0-3$ & $1.99(0.71)$ & $1-4$ & 0.262 \\
\hline Umbilicus & $2.58(0.80)$ & $0.5-4$ & $2.44(0.83)$ & $0.5-4$ & $2.47(0.81)$ & $0.5-4$ & $2.80(0.71)$ & $1-4$ & $0.032^{*}$ \\
\hline Umbilicus $4.5 \mathrm{~cm}$ below & $1.53(0.79)$ & $0-3$ & $1.45(0.89)$ & $0-3$ & $1.37(0.76)$ & $0-3$ & $1.74(0.67)$ & $0-3$ & $0.042^{*}$ \\
\hline \multicolumn{10}{|l|}{ Physical testing } \\
\hline Body weight & $59.9(9.17)$ & $43-87$ & $59.6(7.35)$ & $47-76.8$ & $61.1(10.7)$ & $44.4-83$ & $59.2(9.21)$ & $43-87$ & 0.784 \\
\hline $\begin{array}{c}\text { Waist circumference } \\
\text { Diastasis recti }\end{array}$ & $83.5(7.36)$ & $67-108$ & $83.1(6.97)$ & $67-100$ & 84.1 (8.09) & 71-98 & $83.2(7.11)$ & 69-108 & 0.272 \\
\hline Umbilicus $4.5 \mathrm{~cm}$ above & $0.84(0.52)$ & $0-2$ & $0.84(0.55)$ & $0-2$ & $0.83(0.47)$ & $0-2$ & $0.86(0.54)$ & $0-2$ & 0.411 \\
\hline Umbilicus & $1.51(0.58)$ & $0-3$ & $1.47(0.60)$ & $0.5-3$ & $1.46(0.60)$ & $0-2$ & $1.61(0.55)$ & $0.5-3$ & 0.152 \\
\hline Umbilicus $4.5 \mathrm{~cm}$ below & $0.66(0.53)$ & $0-2$ & $0.65(0.55)$ & $0-2$ & $0.65(0.55)$ & $0-2$ & $0.68(0.51)$ & $0-2$ & 0.674 \\
\hline
\end{tabular}


Table 6: continued

\begin{tabular}{|c|c|c|c|c|c|c|c|c|c|}
\hline \multirow[b]{2}{*}{ Characteristic } & \multicolumn{2}{|c|}{ All } & \multicolumn{2}{|c|}{ DVD-based } & \multicolumn{2}{|c|}{ Internet-base } & \multicolumn{2}{|c|}{ Usual care } & \multirow{2}{*}{$\begin{array}{c}P \\
\text { Comparisor } \\
\text { Among the } \\
\text { Groups }\end{array}$} \\
\hline & $\bar{x}(\mathrm{SD})$ & Range & $\bar{x}(\mathrm{SD})$ & Range & $\bar{x}(\mathrm{SD})$ & Range & $\bar{x}(\mathrm{SD})$ & Range & \\
\hline \multicolumn{10}{|l|}{$\begin{array}{l}\text { At four months } \\
\text { Physical testing }\end{array}$} \\
\hline \multicolumn{10}{|l|}{ Diastasis recti } \\
\hline Umbilicus $4.5 \mathrm{~cm}$ above & $0.46(0.46)$ & $0-2$ & $0.44(0.46)$ & $0-1.5$ & $0.39(0.37)$ & $0-1$ & $0.55(0.51)$ & $0-2$ & 0.323 \\
\hline Umbilicus & $0.92(0.61)$ & $0-3$ & $0.90(0.56)$ & $0-2.5$ & $0.81(0.54)$ & $0-2$ & $1.05(0.70)$ & $0-3$ & 0.257 \\
\hline Umbilicus $4.5 \mathrm{~cm}$ below & $0.43(0.42)$ & $0-1.5$ & $0.42(0.43)$ & $0-1.5$ & $0.39(0.47)$ & $0-1.5$ & $0.44(0.38)$ & $0-1.5$ & 0.857 \\
\hline
\end{tabular}

*Significant differences between the groups $(P<0.05)$ 
Table 7: Uptake exercise eight types at six weeks $(n=99)$ and four months postpartum $(n=58)$ for three groups (mean values)

\begin{tabular}{|c|c|c|c|c|c|c|c|c|c|}
\hline Characteristic & \multicolumn{2}{|l|}{ All } & \multicolumn{2}{|c|}{ DVD-based } & \multicolumn{2}{|c|}{ Internet-base } & \multicolumn{2}{|c|}{ Usual care } & $\begin{array}{c}P \text { Comparison } \\
\text { Among the Groups }\end{array}$ \\
\hline Abdominal breathing exercises & $25.0(12.7)$ & $5-42$ & $27.0(11.3)$ & $8-42$ & $25.2(13.0)$ & $5-42$ & $22.6(14.2)$ & $5-42$ & 0.646 \\
\hline Head and neck exercises & $18.1(13.0)$ & $1-42$ & $18.2(11.7)$ & $1-42$ & $20.3(14.2)$ & $1-41$ & $14.8(12.6)$ & $1-40$ & 0.341 \\
\hline Breast exercises & $19.1(12.9)$ & $1-42$ & $16.9(12.2)$ & $1-42$ & $22.5(13.2)$ & $1-42$ & $19.1(12.9)$ & $1-41$ & 0.238 \\
\hline Gluteus exercises & $18.1(13.6)$ & $1-39$ & $17.5(13.4)$ & $1-39$ & $21.6(13.7)$ & $2-37$ & $14.9(12.5)$ & $1-37$ & 0.257 \\
\hline Vaginal contraction exercises & $20.1(12.5)$ & $1-42$ & $20.0(12.3)$ & $1-42$ & $21.4(12.9)$ & $1-42$ & $19.0(12.7)$ & $1-42$ & 0.771 \\
\hline Abdominal muscle exercises & $15.5(11.3)$ & $1-35$ & $16.7(12.3)$ & $1-35$ & $16.0(10.7)$ & $2-29$ & $12.1(11.1)$ & $2-35$ & 0.551 \\
\hline Knee-to-Chest exercises & $17.6(11.0)$ & $1-35$ & $15.8(12.7)$ & $1-35$ & $21(8.9)$ & $1-29$ & $15.5(10.5)$ & $2-35$ & 0.319 \\
\hline \multicolumn{10}{|l|}{ Four months postpartum } \\
\hline Gluteus exercises & $41.3(23.7)$ & $1-70$ & $38.9(23.4)$ & $1-70$ & $44.4(22.6)$ & $10-70$ & $42.3(28.2)$ & $8-70$ & 0.775 \\
\hline Vaginal contraction exercises & $42.6(22.6)$ & $1-70$ & $41.4(20.7)$ & $5-70$ & $43.0(24.0)$ & $1-70$ & $44.1(25.8)$ & $8-70$ & 0.943 \\
\hline Abdominal muscle exercises & $40.6(23.6)$ & $1-70$ & $39.1(22.9)$ & $1-70$ & $38.3(24.3)$ & $5-70$ & $50.8(25.4)$ & $8-70$ & 0.472 \\
\hline Knee-to-Chest exercises & $40.9(24.9)$ & $1-70$ & $37.2(23.3)$ & $1-70$ & $42.7(25.7)$ & 7-70 & $44.0(28.5)$ & $8-70$ & 0.789 \\
\hline
\end{tabular}


Table 8: Weekly exercise adherence at six weeks $(n=99)$ and four months postpartum $(n=58)$ for three groups (mean values)

\begin{tabular}{|c|c|c|c|c|c|c|c|c|c|}
\hline \multirow[b]{2}{*}{ Characteristic } & \multicolumn{2}{|r|}{ All } & \multicolumn{2}{|c|}{ DVD-based } & \multicolumn{2}{|c|}{ Internet-base } & \multicolumn{2}{|c|}{ Usual care } & \multirow{2}{*}{$\begin{array}{c}\text { P Comparisor } \\
\text { Among the } \\
\text { Groups }\end{array}$} \\
\hline & $\bar{x}(\mathrm{SD})$ & Range & $\bar{x}(\mathrm{SD})$ & Range & $\bar{x}(\mathrm{SD})$ & Range & $\bar{x}(\mathrm{SD})$ & Range & \\
\hline Week 1 & $6.89(4.35)$ & $1-21$ & $5.69(2.56)$ & $1-12$ & $7.66(4.84)$ & $1-21$ & $7.08(4.98)$ & $1-19$ & 0.258 \\
\hline Week 2 & 7.38 (4.52) & $1-28$ & $6.40(3.14)$ & $1-12$ & 7.61 (4.57) & $1-17$ & 8.24 (5.69) & $1-28$ & 0.305 \\
\hline Week 3 & 8.15 (4.98) & $3-28$ & $6.77(2.32)$ & $3-12$ & $9.79(7.06)$ & $3-28$ & 7.66 (3.27) & $3-14$ & 0.065 \\
\hline Week 4 & 7.64 (4.95) & $1-28$ & $6.76(2.32)$ & $2-12$ & 9.35 (7.35) & $1-28$ & $6.61(2.87)$ & $3-14$ & 0.073 \\
\hline Week 5 & 7.30 (5.02) & $2-28$ & $6.40(2.41)$ & $2-12$ & $9.20(7.15)$ & $3-28$ & $5.84(3.07)$ & $2-14$ & $0.032^{*}$ \\
\hline Week 6 & $7.58(4.62)$ & $1-21$ & $7.07(3.80)$ & $2-21$ & $8.53(5.46)$ & $1-20$ & $6.80(4.32)$ & $2-18$ & 0.402 \\
\hline Week 7 & $5.52(2.91)$ & $1-15$ & $5.42(2.71)$ & $1-12$ & 5.87 (3.51) & $1-15$ & $5.30(2.68)$ & $1-10$ & 0.851 \\
\hline Week 8 & $7.63(4.20)$ & $2-20$ & $6.52(1.70)$ & $2-8$ & $9.76(5.87)$ & $2-20$ & $6.75(3.93)$ & $2-16$ & $0.035^{*}$ \\
\hline Week 9 & $6.32(3.06)$ & $1-16$ & $5.27(1.75)$ & $1-7$ & $7.88(4.21)$ & $2-16$ & $6.00(2.14)$ & $2-10$ & $0.025^{*}$ \\
\hline Week 10 & $5.70(2.19)$ & $1-10$ & $5.33(1.73)$ & $2-7$ & $6.05(2.67)$ & $1-10$ & $5.90(2.25)$ & $2-10$ & 0.541 \\
\hline Week 11 & 5.76 (1.65) & $2-8$ & $5.70(1.54)$ & $2-7$ & $5.66(1.78)$ & $2-8$ & 6.10 (1.79) & $3-8$ & 0.785 \\
\hline Week 12 & 5.55 (1.69) & $2-9$ & $5.41(1.61)$ & $2-7$ & $5.44(1.88)$ & $2-9$ & 6.10 (1.59) & $3-8$ & 0.540 \\
\hline Week 13 & 5.82 (1.69) & $2-10$ & $5.40(1.50)$ & $2-7$ & $6.12(1.70)$ & $2-8$ & $6.33(2.06)$ & $3-10$ & 0.274 \\
\hline Week 14 & 5.78 (1.64) & $1-9$ & 5.40 (1.59) & $1-7$ & $6.18(1.75)$ & $2-9$ & $6.00(1.50)$ & $3-7$ & 0.328 \\
\hline Week 15 & $5.53(2.03)$ & $1-10$ & 5.40 (1.59) & $2-7$ & $5.06(2.56)$ & $1-8$ & $6.66(1.65)$ & $4-10$ & 0.154 \\
\hline Week 16 & $5.04(1.53)$ & $1-8$ & $4.86(1.39)$ & $1-6$ & $5.12(1.70)$ & $1-7$ & $5.33(1.65)$ & $3-8$ & 0.724 \\
\hline Week 1 to week 6 & $5.87(4.25)$ & $0.17-22.67$ & $5.47(2.39)$ & $0.83-9.67$ & $6.86(5.79)$ & $0.17-22.67$ & $5.06(3.39)$ & $0.17-14$ & 0.191 \\
\hline Week 7 to week 16 & $5.09(2.33)$ & $0.10-8.80$ & $4.86(2.04)$ & $0.10-7.10$ & $5.62(2.44)$ & $1.5-8.80$ & $4.78(2.73)$ & $0.8-8.8$ & 0.491 \\
\hline
\end{tabular}

* Significant differences between the groups $(P<0.05)$ 
Table 9: Postpartum exercise of completion rate at six weeks $(n=128)$ and four months postpartum $(n=105)$ for three group

All DVD-based Internet-base Usual care

$\begin{array}{lllll}\text { Characteristic } & \text { N (\%) } & \text { N (\%) } & \text { N (\%) } & \text { N (\%) }\end{array}$

Six weeks postpartum

$\begin{array}{lllll}\text { Yes } & 99(77.3) & 33(25.8) & 37(28.9) & 29(22.7) \\ \text { No } & 29(22.7) & 10(7.8) & 5(3.9) & 14(10.9)\end{array}$

Four months postpartum

$\begin{array}{lllll}\text { Yes } & 58(55.2) & 26(24.8) & 19(18.1) & 13(12.4) \\ \text { No } & 47(44.8) & 9(8.6) & 13(12.4) & 25(23.8)\end{array}$




\section{Adverse Events}

There were no adverse events associated with this trial. 